\title{
Internal structure of voicing categories in early infancy
}

\author{
JOANNE L. MILLER \\ Northeastern University, Boston, Massachusetts \\ and \\ PETER D. EIMAS \\ Brown University, Providence, Rhode Island
}

\begin{abstract}
It is well established that young infants process speech in terms of perceptual categories that closely correspond to the phonetic categories of adult language users. Recently, Kuhl (1991) has provided evidence that this correspondence is not limited to the region of category boundaries: At least by 6-7 months of age, vowel categories of infants, like those of adults, have an internal perceptual structure. In the current experiments, which focused on a consonantal contrast, we found evidence of internally structured categories in even younger infants-3-4 months of age. The implications of these findings for the nature of the infant's earliest language-universal categories are discussed, as is the role of exposure to the native language in shaping these categories over the course of development.
\end{abstract}

One of the fundamental issues in speech perception is how listeners map the continuously varying signal of speech onto the phonetic categories of their language. Much of the research on phonetic categorization over the years has focused on the boundaries between phonetic categories, addressing such issues as why boundaries fall where they do along given acoustic continua, what kinds of contextual factors play a role in determining boundary location, and the effects of specific linguistic experience in altering boundary location over the course of development (see, e.g., Repp \& Liberman, 1987). This emphasis on boundaries derived in part from the much-studied phenomenon of categorical perception, which emphasized the relative difficulty experienced by listeners in discriminating members of a given phonetic category compared with the ease in discriminating tokens that cross a category boundary (Repp, 1984; Studdert-Kennedy, Liberman, Harris, \& Cooper, 1970).

It has become increasingly clear, however, that phonetic categories are much richer in form than this emphasis on boundaries would suggest. It is now known that listeners can discriminate stimuli from a given category with a relatively high degree of accuracy, given specific stimulus and task conditions (Carney, Widin, \& Viemeister, 1977; Samuel, 1977; see also Macmillan, Goldberg, \& Braida, 1988). Moreover, and of particular relevance for the current research, there is a growing literature indicating that phonetic categories have a graded internal struc-

This research was supported by NIH Grant DC 00130 to J.L.M.and NIH Grant HD 05331 to P.D.E. We thank J. Shepp, L. Kilpatrick, and $\mathrm{L}$. Yarzab for their assistance in testing subjects, and J. Mertus for making available the BLISS system for testing infants. Correspondence should be addressed to J. L. Miller, Department of Psychology, Northeastern University, Boston, MA 02115 (e-mail: jlmiller@neu.edu). ture. That is to say, stimuli within a category are not only discriminable from one another, but are perceived as varying in category goodness, with some members of a category perceived as better exemplars, or more prototypical, than others (Oden \& Massaro, 1978). Thus phonetic categories have an internal structure that is reminiscent of the kind of structure seen for nonspeech perceptual/ cognitive categories (Medin \& Barsalou, 1987; Nosofsky, 1988; Rosch, 1978). The internal structure of phonetic categories is evident in tasks requiring the overt judgment of category goodness (Kuhl, 1991; Massaro \& Cohen, 1983; Miller \& Volaitis, 1989; Samuel, 1982), as well as in tasks that measure the functional salience of category members by assessing, for example, dichotic competition (Miller, 1977; Repp, 1977), selective adaptation (Miller, Connine, Schermer, \& Kluender, 1983; Samuel, 1982), and discrimination (Kuhl, 1991). ${ }^{1}$

The issue we address in the present research concerns the developmental origins of this internal category structure. It has been known for many years that young, prearticulate infants process speech in terms of categories (for a review, see Jusczyk, 1995). Much of the evidence for infant categorization comes from discrimination studies showing that infants, like adults, are better at discriminating stimuli from different (adult) phonetic categories than stimuli from the same category. Interestingly, it has also been shown that the boundaries of infant categories, like those of adults, are sensitive to acoustic-phonetic contextual factors and to the multiple properties of the acoustic signal that specify phonetic contrasts (Miller \& Eimas, 1983 ). Thus, the evidence indicates that very young infants partition the phonetically relevant acoustic space in terms of perceptual categories that are well positioned to form the precursors of adult phonetic categories. These initial "language-universal" categories of the infant are then modified through exposure to the native language to 
become the language-specific categories of adult language users (for reviews, see Best, 1994; Werker, 1994).

Recently, Kuhl and her colleagues (Grieser \& Kuhl, 1989; Kuhl, 1991; see also Polka \& Werker, 1994) have provided evidence that the infant's categories, like the adult's, are internally structured. The evidence comes from studies of the vowel category $/ \mathrm{i} /$ in infants $6-7$ months of age. The stimuli used in the studies were synthesized to simulate a male speaker and differed systematically from each other in their first two formant frequencies. These tokens were judged by adults to vary in category goodness, with some stimuli perceived as better exemplars of /i/ than others.

The infants were tested with a head-turn conditioning procedure. In this procedure, infants are presented a repeating background stimulus and are assessed on their ability to discriminate a change from the background to a different stimulus. For half of the infants tested (the prototype group) the background stimulus was a good exemplar of the /i/ category, whereas for the other half (the nonprototype group), the background stimulus was a poor exemplar of this category. ${ }^{2}$ The major finding was that discrimination was poorer in the prototype group than in the nonprototype group. In other words, infants who heard the prototype as the background stimulus generalized more to surrounding stimuli than did infants who heard the nonprototype as the background stimulus. A comparison group of adults showed a similar effect. Kuhl and her colleagues interpreted these findings in terms of a "perceptual magnet effect." The basic idea is that for infants as well as adults, the category is structured in terms of an internal prototype, and the prototype acts as a "magnet," functionally pulling surrounding stimuli toward itself in psychological space. This effectively shrinks the perceptual space around the prototype, compared with the space around the nonprototype, making discrimination in the prototype region more difficult. Subsequent research on adults using multidimensional scaling based on similarity ratings (Iverson \& Kuhl, 1995) has provided support for this idea, in that stimulus tokens located in the region of the prototype are perceptually closer together than are tokens located at the outskirts of the category.

Kuhl's infant findings indicate that, at least by 6 months of age, vowel categories are internally structured. The purpose of the present study was to extend this research in three ways. First, we asked whether the infant's consonant categories are also internally structured. Second, we asked whether such structure is evident even earlier in life than 6 months of age. Finally, we used a somewhat different strategy to assess category structure as a way of providing converging evidence for its existence. We consider each of these points in turn.

\section{Consonant Categories}

There has been a long history in the field concerning a possible distinction in the way in which listeners process consonants and vowels. In particular, some have argued that consonants are generally perceived "more cat- egorically" than vowels, so that it is easier for listeners to distinguish variants of a vowel category than to distinguish variants of a consonant category. Vowel discrimination thus appears to be less constrained by category identity than is consonant discrimination (for review, see Repp, 1984). Because the degree of within-category discrimination depends on numerous stimulus and task factors, it is difficult to equate "consonants" and "vowels" on all the relevant parameters, and thus a direct test of this notion is not at hand. Nonetheless, there is some evidence in support of the processing distinction (see, e.g., Schouten \& van Hessen, 1992; see also Macmillan et al., 1988). Moreover, the stimuli used in the Kuhl studies were rather long steady-state vowels ( $500 \mathrm{msec}$ in duration) that would be expected to yield evidence of within-category discrimination in infants (Swoboda, Morse, \& Leavitt, 1976; cf. Swoboda, Kass, Morse, \& Leavitt, 1978). Our question was whether we could also find evidence of internal category structure in infants for a voiceless stop consonant category that varied in voice-onset-time (VOT), an articulatory/acoustic parameter that serves to distinguish the voiceless stops from their voiced counterparts $(/ \mathrm{p} /$ from $/ \mathrm{b} /, / \mathrm{t} /$ from $/ \mathrm{d} /, / \mathrm{k} /$ from $/ \mathrm{g} /$ ). Although the existing literature suggests that within-category discrimination for such categories is limited in infants (e.g., Eimas, Siqueland, Jusczyk, \& Vigorito, 1971), there has been no direct test of within-category structure, per se-that is, no test of whether there are differences in discriminability related to differences in category goodness.

The phonetic category we chose to study was the syllable-initial voiceless stop consonant (specifically, $/ \mathrm{k} /$ and $/ \mathrm{p} /$ ). Within-category variation was specified by a change in VOT. As noted above, early research has shown that infants, like adults, categorize stimuli varying in VOT, so that they readily discriminate tokens falling across the voiced-voiceless boundary, but have considerable difficulty discriminating stimuli from a single category, that is, two voiced or two voiceless stimuli (Eimas et al., 1971). Subsequent research has shown, however, that at least for adults, within-category discrimination can be quite good under certain task conditions (e.g., Carney et al., 1977; Pisoni \& Tash, 1974; see Repp, 1984, for a review). Moreover, and of particular relevance to the present work, it is now well established that the voiceless category in adults has a graded internal structure. Adults perceive some tokens of the voiceless category as better exemplars than others (Miller \& Volaitis, 1989; Volaitis \& Miller, 1992), and these differences are correlated with differences in the functional salience of the stimuli in tasks such as speeded goodness judgments (Wayland \& Miller, 1992) and selective adaptation (Miller et al., 1983; Samuel, 1982). Moreover, multidimensional scaling based on similarity ratings has shown that for this category, as for vowel categories, stimulus tokens located near the region of the prototype are perceptually closer than are those located away from the prototype (Davis \& Kuhl, 1994). In the present study, we asked whether the infant's voiceless stop category also has internal perceptual structure. 


\section{Younger Infants}

Kuhl and her colleagues tested infants 6 -7 months of age. In the present study, we tested younger infants, 34 months of age. Testing younger infants is important in its own right for determining when within-category structure first appears during the course of development. Moreover, there is a distinction between consonants and vowels that makes it particularly interesting to test for structure in consonant categories at this early age. The literature suggests that infants begin life with language-universal categories and that these categories are altered by exposure to the native language during the course of development to become the language-specific categories of adults. This modification takes a variety of forms. For example, category boundaries are shifted along an underlying acoustic continuum so as to change the precise mapping between acoustic continuum and perceptual category to be in line with that of the native language, and some boundaries are eliminated altogether as they are in the native language, so that distinctions that were readily discriminable in early infancy are no longer differentiated by older infants or adults (for reviews and discussion of possible mechanisms underlying these changes, see Best, 1994; Jusczyk, 1995; Kuhl, 1993; Werker, 1994).

Of particular importance for the present study is the finding that linguistic experience seems to have its effect earlier for vowel categories than for consonant categories. The impact of exposure to the native language is seen for consonant categories near the end of the 1st year of life (Werker \& Lalonde, 1988; Werker \& Tees, 1984). Recent evidence indicates, however, that native-language exposure has an influence on vowel categories as young as 6 months of age. Moreover, this influence involves internal category structure. Specifically, in a test of the perceptual magnet effect, Kuhl, Williams, Lacerda, Stevens, and Lindblom (1992) found that which of a pair of vowels showed poorer discrimination from surrounding vowels depended on its prototypicality in the native language of the infant. American infants showed poorer discrimination for a prototypical English vowel than for a prototypical Swedish vowel (which was a nonprototypical vowel in English), whereas Swedish infants showed the converse pattern. Additional evidence that language experience influences vowel perception by 6 months of age is provided by Polka and Werker (1994); their data also provide at least indirect confirmation of internally structured vowel categories shaped by the native language at this age.

These data indicate that by 6 months of age, the infant's vowel categories have been shaped by linguistic experience, and are structured. What these findings do not reveal is whether the initial, language-universal vowel categories of the infant are also structured, with language exposure serving to fine-tune this structure in accord with the distributional properties of the native language, or whether these earliest categories are unstructured, with internal structure developing only as the infant is exposed to exemplars of the native language (Kuhl, 1993). Of course this fundamental issue applies not only to vowels, but also to consonants. We know that adult consonant categories are internally structured, but when does this structure arise? In particular, does it arise only in the course of listening to a particular language, or are the earliest, language-universal consonant categories of the infant themselves internally structured?

As a first attempt to address this issue, we asked whether a consonantal voicing category would show evidence of internal structure in 3- to 4-month-old infants. As noted earlier, the literature suggests that language exposure does not influence the location of boundaries between consonant categories until the end of the 1st year of life. Of special relevance is the finding that at 4-6 months of age, native-language exposure has apparently not yet altered the perception of voicing categories. At this age, the location of the voiced-voiceless boundary has not yet moved in accord with its location in the parental language (Lasky, Syrdal-Lasky, \& Klein, 1975), and even at $6-8$ months of age, infants still discriminate nonnative voicing contrasts that nonnative adults find difficult (Werker, Gilbert, Humphrey, \& Tees, 1981). Thus, if we find internally structured voicing categories as early as 3-4 months of age, we will have at least preliminary evidence that the initial, language-universal consonant categories of the infant may themselves be internally structured, with exposure to the native language serving to fine-tune this structure (as well as the boundaries between categories) over the course of development.

\section{Testing Paradigm}

We used a somewhat different way of testing for internal structure in infancy than was used by Kuhl (1991; Kuhl et al., 1992; Grieser \& Kuhl, 1989). Kuhl's procedure involves comparing discrimination of stimuli in two regions of category space, stimuli near the prototype and stimuli far from the prototype. As noted above, the results indicate that there is an asymmetry in within-category discrimination, so that it is more difficult to discriminate stimuli that differ from the prototype than it is to discriminate stimuli that differ from the nonprototype. This is assumed to be due to a warping of the perceptual space that effectively reduces the perceptual distances among stimuli near the prototype (and thus makes discrimination in that region more difficult), and is taken as evidence that the category itself is internally structured vis-à-vis the prototype.

There is another kind of asymmetry, however, that has also been taken as evidence for graded internal structure. This is an asymmetry in the perceived similarity of two stimuli from a given category that depends on which of the two stimuli, the prototypical category exemplar or the nonprototypical exemplar, serves as the standard for the similarity judgment (Rosch, 1975). The empirical finding is that a nonprototypical exemplar is judged to be more similar (perceptually closer) to the category prototype than the category prototype is to the nonprototypical exemplar. For example, a nonprototypical line orientation (e.g., an almost vertical line) is judged to be more 
similar to a prototypical line orientation (e.g., a vertical line) than the reverse (Rosch, 1975; see also Krumhansl, 1978, 1979; Tversky, 1977). Thus, two stimuli from a given category are perceived to be more similar when the prototype serves as the standard for the similarity judgment than when the nonprototype serves as the standard.

Our infant study was based on this idea, with the assumption that differences in perceptual similarity would be revealed in ease of discrimination: the more perceptually similar the two stimuli, the more difficult the discrimination. More specifically, we reasoned that if the infant's categories are internally structured with respect to a category prototype, then two stimuli from within a given category, a prototype and a nonprototype, should be perceived as more similar - and hence be less discriminable - when the prototype serves as the standard for discrimination than when the nonprototype serves as the standard. We tested this prediction using a visual habituation procedure (Best, McRoberts, \& Sithole, 1988; Eimas \& Miller, 1992; Horowitz, 1975), which is analogous to the high-amplitude-sucking habituation procedure originally introduced by Eimas et al. (1971) for testing speech perception in infants. In the visual habituation procedure, infants are first familiarized with one of two speech stimuli, which serves as the standard. During this familiarization phase, the speech stimulus is presented contingent on the infant's looking at a picture. After a criterion decline in looking time has been met (i.e., habituation has occurred), the second speech stimulus is presented, again contingent on looking at the picture. An increase in looking time (dishabituation) relative to a control group for which the sound does not change is taken as evidence for discrimination. In our study, the stimulus pairs consisted of a prototypical exemplar of $/ \mathrm{ki} /$ paired with a nonprototypical exemplar of $/ \mathrm{ki} /$ (Experiment 1$)$ or a prototypical exemplar of /pi/ paired with a nonprototypical exemplar of /pi/ (Experiment 2). In each case, discrimination was assessed both when the prototype was the familiarization stimulus (the standard) and when the nonprototype was the familiarization stimulus (the standard). If infants' voicing categories are internally structured with respect to a category prototype, it should be harder to discriminate the pair when the prototypical $/ \mathrm{ki} /$ (or $/ \mathrm{pi} /$ ) serves as the familiarization stimulus than when the nonprototypical $/ \mathrm{ki} /$ (or $/ \mathrm{pi} /$ ) serves as the familiarization stimulus. However, if the infant's voicing categories are unstructured at this early age, discrimination should be the same regardless of which stimulus is presented during familiarization.

\section{EXPERIMENT 1}

The first experiment focused on variants of the voiceless stop category $/ \mathrm{k} /$, with the variants differing from each other in VOT. Two pairs of stimuli were tested. In each case, one member of the pair was a prototypical exemplar of $/ \mathrm{k} /$ and the other was a nonprototypical exemplar of $/ \mathrm{k} /$ according to adult goodness ratings (see below). In order to increase our chances of obtaining evidence for within- category discrimination, we used within-pair VOT differences that were larger than those used in earlier studies of infant voicing perception (Eimas et al., 1971; Miller \& Eimas, 1983). The two stimulus pairs differed from each other in that for one pair, the nonprototype had a shorter VOT value than the prototype, whereas for the other pair, the nonprototype had a longer VOT value.

If the infant's voiceless category is internally structured with respect to the prototype, discrimination for a given pair should be better when the familiarization stimulus (the standard) is the nonprototypical $/ \mathrm{k} /$ than when it is the prototypical $/ \mathrm{k} /$. Furthermore, if the asymmetry is due to the prototype/nonprototype status of the standard, per se, and not to a possible asymmetry in discriminating short from long, versus long from short, VOT values, this asymmetry should be found for both stimulus pairs-that is, both when the nonprototype has a shorter VOT value than the prototype and when it has a longer VOT value than the prototype.

\section{Method}

Subjects. The subjects were 128 infants, $3-4$ months of age ( $M=3.35$ months; $S D=.48$ months). There were 56 females and 72 males. An additional 207 infants did not complete the study because (1) they were fussy $(n=94),(2)$ they failed to habituate or to elicit the sound by looking at the picture $(n=92)$, or (3) the computer malfunctioned $(n=21)$. The vast majority of infants were Caucasian and from middle- and upper-middle-class homes situated in the greater Providence area. All infants were recruited by obtaining their mothers' permission to participate shortly after their birth at Women and Infants Hospital of Rhode Island.

Sixteen infants were assigned to each of the eight treatment groups that resulted from the factorial combination of conditions (experimental vs. control), familiar stimulus (prototype vs. nonprototype), and stimulus pair (VOT $=65 / 110$ vs. $100 / 200 \mathrm{msec}$ ). The assignment of infants was as nearly random as possible given the high attrition rate and the attempt to balance gender.

Stimuli. The stimuli were four five-formant synthetic consonantvowel (CV) syllables from the $325-\mathrm{msec}$ velar series described by Volaitis and Miller (1992) and used originally to investigate the internal category structure of voiceless stop consonants in adult listeners. The original velar series ranged from $/ \mathrm{gi} /$ to $/ \mathrm{ki} /$ to a breathy, exaggerated version of $/ \mathrm{ki} /$. The change across the series was specified by a change in VOT. The change in VOT was accomplished by cutting back first-formant energy and replacing the periodic source of the synthesizer with a noise source during the cutback, so as to simulate aspiration. Each stimulus was $325 \mathrm{msec}$ in duration and consisted of a 10 -msec release burst followed by $5 \mathrm{msec}$ of silence, a 45-msec transition segment ( $F 1$ transition duration was $35 \mathrm{msec}$ ), and a steady-state segment appropriate for the vowel $/ \mathrm{i} /$. Details of stimulus construction can be found in Volaitis and Miller (1992).

The four syllables selected for the present experiment had VOT values of $65,100,110$, and $200 \mathrm{msec}$. Adults in the Volaitis and Miller (1992) experiment judged the 100- and 110-msec VOT stimuli to be good exemplars of $/ \mathrm{ki} /$ and the $65-$ and $200-\mathrm{msec}$ stimuli to be poor exemplars of $/ \mathbf{k i} /$. More specifically, in a three-choice identification task requiring identification of the syllables as $/ \mathrm{gi} /$, $/ \mathrm{ki} /$, or a breathy, exaggerated version of $/ \mathrm{ki} /$ (labeled $* / \mathrm{ki} /$ ), adults identified the $65-\mathrm{msec}$ stimulus as $/ \mathrm{ki} /$ or $* / \mathrm{ki} /$ on over $85 \%$ of the trials and labeled the other three stimuli as $/ \mathrm{ki} /$ or $* / \mathrm{ki} /$ on over $97 \%$ of the trials. Thus all four stimuli were perceived as variants of the $/ \mathrm{k} /$ category. According to data from a category goodness rating task, both the 100- and 110-msec stimuli fell within the "best-exemplar range" for $/ \mathrm{ki} /(72.9$ - to $122.2-\mathrm{msec}$ VOT), whereas the other two 
stimuli fell outside of this range. Thus the four stimuli consisted of two good exemplars of $/ \mathrm{ki} /(100$ - and 110-msec VOT), one poor exemplar of $/ \mathrm{ki} /$ with a shorter VOT value $(65 \mathrm{msec})$, and one poor exemplar of $/ \mathrm{ki}$ / with a longer VOT value $(200 \mathrm{msec})$. The $100-$ and 110 -msec stimuli will be referred to as the category prototypes; the 65- and 200-msec stimuli will be referred to as the nonprototypes. For testing, the stimuli with VOT values of 65 and $110 \mathrm{msec}$ were paired together, as were the stimuli with VOT values of 100 and 200 msec. $^{3}$

Apparatus. The stimuli were digitally transferred and stored on an IBM PC AT, which controlled the execution of the experiment by means of programs developed by John Mertus at Brown University as part of the BLISS system. The stimuli were presented to the infant by means of a mini Advent speaker. The intensity of the comparable vocalic portions was set to remain constant across stimuli; this necessarily resulted in a slight decrease in the overall level of the syllable as VOT increased. The overall levels (dBA SPL, measured at the infant's head) were approximately $78,76,76$, and 74 for the 65-, 100-, 110-, and 200-msec VOT stimuli, respectively.

The speaker was located approximately $65 \mathrm{~cm}$ from the infant's head and immediately above a stationary picture of a lighted woman's face. Just below the woman's jaw was a hole in a black-painted panel through which a permanently mounted TV camera recorded the baby's face. The image of the baby was displayed on a monitor situated in a separate room. The experimenter in the room with the monitor viewed the baby and pressed a button on a response panel whenever the picture of the woman was centered on the infant's corneas. The button was released when the baby looked away from the woman. Pressing the button initiated a repetitive presentation of one of the speech stimuli, and releasing it stopped stimulus presentation when the stimulus being presented ended.

Procedure. As noted above, a visual habituation procedure was used in this experiment (Best et al., 1988; Eimas \& Miller, 1992; Horowitz, 1975). Each infant was placed in a reclining infant's seat in a dimly lit, sound-attenuated room facing the lighted picture of a woman's face and the speaker. The picture, which was $15.4 \times$ $14.7 \mathrm{~cm}$, was located $61 \mathrm{~cm}$ from the infant's eyes and subtended angles of $14^{\circ} 3^{\prime}$ vertically and $13^{\circ} 30^{\prime}$ horizontally. After the infant was comfortably settled by the first experimenter, the experiment began. The first experimenter, who was seated to the side of the infant during the entire experiment, listened to recorded music over earphones so that she could not hear the speech stimuli being presented to the infant. On each trial, a second experimenter, who was naive with respect to experimental condition, observed the infant's face on a monitor in a separate room. When this experimenter judged that the infant was observing the face as evidenced by seeing the face centered on the infant's corneas, a button was pressed and held down. The experimenter's response resulted in one of two speech stimuli being presented at the rate of one stimulus per second. The experimenter released the button whenever the infant looked away from the picture, stopping stimulus presentation when the stimulus being played ended. The second experimenter could, by turning her head briefly, observe a second monitor that displayed the trial number and shift period (pre or post); she would thus know when to terminate the experiment. Interexperimenter reliability for this procedure for a variety of dependent measures has been shown to be quite high in our laboratory, being between .84 and .96 (Eimas \& Miller, 1992).

A trial was defined as an infant observing the picture for a minimum of 4 , not necessarily consecutive, sec, followed by a continuous period of at least $1 \mathrm{sec}$ during which the infant looked away from the picture. If the infant looked away from the picture for less than $1 \sec$ (i.e., during a trial), the clock recording total observation time for the trial stopped as soon as the infant stopped fixating the picture, and then resumed when the infant again fixated the picture. The familiarization stimulus was presented until the mean fixation time for the final three consecutive trials was $50 \%$ or less than the mean fixation time for the first three trials. There was thus a minimum of six familiarization (preshift) trials. When the familiarization criterion was reached, the six-trial test (postshift) phase began. In the experimental conditions, the infants heard a different stimulus, whereas infants in the control conditions heard the same stimulus as they had during familiarization.

\section{Results and Discussion}

Familiarization. Table 1 shows the mean looking times as a function of trials, conditions, and whether the familiar stimulus was a prototypical or nonprototypical category exemplar. The results, which have been averaged across the two stimulus pairs (65/110 and 100/200 msec) because the pattern of results was essentially the same for both pairs, indicate no substantial differences in familiarization across groups. This was confirmed by separate analyses of variance (ANOVAs) - conditions (experimental vs. control) $\times$ familiar stimulus (prototype vs. nonprototype $) \times$ stimulus pair $(65 / 110 \mathrm{vs} .100 / 200 \mathrm{msec})-$ which were performed on individual mean scores for the first three and last three trials of familiarization. In both analyses, there were no reliable effects as a function of conditions, familiar stimulus, or stimulus pair $[F(1,120)<$ $1.50, p>.10$, in all instances]. There was one significant interaction, the conditions $\times$ stimulus pair interaction $[F(1,120)=4.13, p<.05]$ in the analysis for the first three familiarization trials. The mean looking score for the control condition for infants familiarized with stimuli having VOT values of 65 or $110 \mathrm{msec}$ was significantly lower than that for any of the other combinations

Table 1

Mean Looking Times (in Seconds) and Standard Deviations for Experiment 1

\begin{tabular}{|c|c|c|c|c|c|c|c|c|}
\hline \multirow[b]{3}{*}{ Familiar Stimulus } & \multicolumn{4}{|c|}{ Preshift Trials } & \multirow{2}{*}{\multicolumn{2}{|c|}{$\frac{\text { Postshift Trials }}{\text { First Three }}$}} & \multirow{2}{*}{\multicolumn{2}{|c|}{ Difference* }} \\
\hline & \multicolumn{2}{|c|}{ First Three } & \multicolumn{2}{|c|}{ Last Three } & & & & \\
\hline & $M$ & $S D$ & $M$ & $S D$ & $M$ & $S D$ & $M$ & $S D$ \\
\hline \multicolumn{9}{|c|}{ Experimental Subjects } \\
\hline Nonprototype & 27.19 & 12.97 & 8.22 & 3.93 & 13.32 & 7.35 & 5.10 & 5.70 \\
\hline Prototype & 26.37 & 16.51 & 8.55 & 3.23 & 9.66 & 3.31 & 1.11 & 3.89 \\
\hline \multicolumn{9}{|c|}{ Control Subjects } \\
\hline Nonprototype & 25.10 & 17.51 & 7.86 & 3.31 & 9.82 & 4.73 & 1.96 & 4.94 \\
\hline Prototype & 23.29 & 13.44 & 8.59 & 4.05 & 9.72 & 3.96 & 1.13 & 3.93 \\
\hline
\end{tabular}

*The mean looking times on the first three postshift trials minus the mean times on the last three preshift trials. 
of conditions and stimulus pairs. In that this effect was not present during the final three trials of familiarization, it did not influence the difference scores (see below). Moreover, the low scores in the control condition could have had no effect on the comparison of greatest importance, namely that between experimental infants familiarized with a prototype and experimental infants familiarized with a nonprototype.

Difference scores. Difference scores were computed by subtracting the mean looking times averaged across the last three trials of familiarization from the mean looking times averaged across the first three postshift trials. ${ }^{4}$ These are shown averaged across the two stimulus pairs $(65 / 110$ and $100 / 200 \mathrm{msec}$ ) in Table 1, and separately for the two pairs in Table 2. As the tables show, infants in the experimental condition who were familiarized with a nonprototype produced, as was predicted, the largest mean difference score. An ANOVA on these scores-conditions (experimental vs. control) $\times$ familiar stimulus (prototype vs. nonprototype $) \times$ stimulus pair $(65 / 110$ vs. $100 /$ $200 \mathrm{msec}$ ) - showed a significant effect for familiar stimulus $[F(1,120)=8.25, p<.01]$ : Infants familiarized with a nonprototypical stimulus had greater difference scores than did those familiarized with a prototypical stimulus. The effect of conditions only approached significance $[F(1,120)=3.46, p<.10]$, as did the expected interaction between familiar stimulus and conditions $[F(1,120)=$ $3.56, p<.10]$. However, as noted above (and see the individual comparisons described below), the pattern of data showed that only the infants in the experimental condition who were familiarized with a nonprototype produced a large increase in their looking response. The effect of stimulus pair and all other interactions were not significant $[F(1,120)<1, p>.10$, in all cases $]$.

Individual comparisons were performed on the difference scores shown in Table 1 to test specific predictions. ${ }^{5}$ The most important comparison confirmed the prediction that experimental infants who were familiarized with a nonprototype would show a larger difference score than would experimental infants familiarized with a prototype $(p<.01)$. In the next two comparisons, we tested whether either or both of the experimental groups showed evidence of discrimination; these tests involved comparison of experimental and appropriate control groups. The difference score for the experimental infants familiarized

Table 2

Mean Difference Scores (See Text) and Standard Deviations for Experiment 1

\begin{tabular}{lllcc}
\hline $\begin{array}{c}\text { Stimulus } \\
\text { Pair }\end{array}$ & Subjects & Familiar & & \\
\hline $65 / 110$ & experimental & nonprototype & 5.46 & 6.47 \\
& & prototype & 1.66 & 4.50 \\
& control & nonprototype & 2.05 & 3.81 \\
& & prototype & 1.36 & 2.62 \\
$100 / 200$ & \multirow{2}{*}{ experimental } & nonprototype & 4.74 & 5.09 \\
& & prototype & .56 & 3.22 \\
& control & nonprototype & 1.87 & 5.98 \\
& & prototype & .90 & 4.99 \\
\hline
\end{tabular}

Note-Values for stimulus pairs are VOTs, in milliseconds. with a nonprototype was reliably larger than that of their control infants $(p<.01)$, but this was not the case for the experimental infants familiarized with a prototype $(p>$ .10 ). Thus, only the infants familiarized with a nonprototypical stimulus showed evidence of discrimination.

It is important that the results described above hold for both sets of stimuli; that is, they must hold regardless of whether the nonprototype lies nearer or farther from the category boundary than does the prototype. The difference scores for the two stimulus pairs $(65 / 110$ and $100 /$ $200 \mathrm{msec}$ ), shown in Table 2, indicate that this is indeed the case. Individual comparisons showed that the difference scores for experimental subjects familiarized with a nonprototype and for infants familiarized with a prototype were significantly different, for each stimulus pair (for $65 / 110 \mathrm{msec}, p<.05$; for $100 / 200 \mathrm{msec}, p<.025$ ). In addition, experimental infants who had been familiarized with a nonprototype showed a greater increase in their looking times than did their comparable controls for both stimulus pairs $(p<.025$ for the $65 / 110-\mathrm{msec}$ pair and $p<.05$ for the $100 / 200$-msec pair), but this was not true for infants familiarized with a prototype for either stimulus pair ( $p>.10$ in each case). Thus, the pattern of results averaged across stimulus pairs was replicated for each pair of stimuli considered separately.

Finally, analyses of the difference scores for the last three postshift trials revealed no reliable differences, although the mean difference score for the experimental group familiarized with a nonprototype was again the largest difference score and approached being significantly different from the experimental group familiarized with a prototype $(p<.10)$.

In summary, we obtained the predicted asymmetry in dishabituation for the experimental infants: Dishabituation was greater when the familiar stimulus--the standard-was a nonprototypical exemplar of the category than when it was a prototypical exemplar of the category. Moreover, this was true both when the nonprototype had a shorter VOT value than the prototype and when it had a longer VOT value. Interestingly, the asymmetry in dishabituation was sufficiently strong that only the infants familiarized with a nonprototype showed evidence of discrimination compared with their control group. Overall, the asymmetries in performance for the two experimental groups provide evidence that the infant's voiceless $/ \mathrm{k} /$ category has a graded internal structure, as early as 3-4 months of age.

\section{EXPERIMENT 2}

The purpose of Experiment 2 was to replicate these findings using another voiceless stop category, $/ \mathrm{p} /$. Ideally, we would have again tested two stimulus pairs, one with the nonprototype located toward the voiced-voiceless category boundary and the other with the nonprototype located beyond the prototype in the other direction. However, we were only able to test infants with a single pair of stimuli, a prototypical exemplar (80-msec VOT) and a nonprototypical exemplar with a longer VOT value (180- 
msec VOT). It was not possible to use a nonprototypical, within-category stimulus in the direction of the voicedvoiceless boundary because for the /p/ category, the distance between the lower edge of the "best-exemplar range" and the voiced-voiceless boundary is less than $5 \mathrm{msec}$ (see Volaitis \& Miller, 1992).

\section{Method}

Subjects. There were 64 subjects, 3 - to 4 -month-old infants ( $M=3.36$ months; $S D=.44$ months), drawn from the same population as the infants of Experiment 1 and recruited in the same manner. There were 29 females and 35 males. An additional 82 infants failed to complete the experimental procedure because (1) they were fussy $(n=34)$, (2) they did not habituate or elicit the sound by looking at the picture $(n=40)$, or (3) the computer malfunctioned $(n=8)$.

Sixteen infants were assigned to each of the four treatment groups formed from the factorial combination of conditions (experimental vs. control) and familiar stimulus (prototype vs. nonprototype). The assignment of infants was as nearly random as possible given the high attrition rate and the attempt to balance gender.

Stimuli. The stimuli were two five-formant synthetic CV syllables from the 325-msec labial series described by Volaitis and Miller (1992). The original labial series ranged from / bi/ to / $\mathrm{pi} /$ to a breathy, exaggerated version of $/ \mathrm{pi} /$. The change in VOT across the series was accomplished by cutting back first-formant energy and replacing the periodic source of the synthesizer with a noise source during the cutback so as to simulate aspiration. Each stimulus was $325 \mathrm{msec}$ in duration and consisted of a $5-\mathrm{msec}$ release burst followed by $5 \mathrm{msec}$ of silence, a 45 -msec transition segment ( $F 1$ transition duration was $20 \mathrm{msec}$ ), and a steady-state segment appropriate for the vowel / 1 /. Details of stimulus construction can be found in Volaitis and Miller (1992).

Two syllables were selected for the current experiment, those with VOT values of 80 and $180 \mathrm{msec}$. Adults in the Volaitis and Miller (1992) experiment judged the $80-\mathrm{msec}$ VOT stimulus to be a good exemplar of /pi/ and the $180-\mathrm{msec}$ stimulus to be a poor exemplar of $/ \mathrm{pi} /$. More specifically, in a three-choice identification task requiring identification of the syllables as $/ \mathrm{bi} /, / \mathrm{pi} /$, or a breathy, exaggerated version of $/ \mathrm{pi} /($ labeled $* / \mathrm{pi} /$ ), adults identified each of the stimuli as $/ \mathrm{pi} /$ or $* / \mathrm{pi} /$ on $100 \%$ of the trials. Thus both stimuli were perceived as variants of the / $\mathrm{p} /$ category. According to data from a category goodness rating task, the $80-\mathrm{msec}$ stimulus fell within the best-exemplar range for / pi/ (49.1- to 103.6-msec VOT), whereas the other stimulus fell outside of this range. Thus the two stimuli consisted of one good exemplar of /pi/ ( $80-\mathrm{msec}$ VOT), which will be referred to as the category prototype, and one poor exemplar of $/ \mathrm{pi}$ / with a longer VOT value $(180 \mathrm{msec})$, which will be referred to as the nonprototype.
Apparatus and Procedure. The apparatus and procedure were identical to those described in Experiment 1. As in Experiment 1, the stimuli were presented in such a way that the intensity of the comparable vocalic portions remained constant. This resulted in overall levels (dBA SPL, measured at the infant's head) that were approximately 78 and 75 for the 80 - and $180-\mathrm{msec}$ VOT stimuli, respectively.

\section{Results and Discussion}

Familiarization. The mean looking times are shown in Table 3. As can be seen, the groups showed comparable performance during familiarization. Separate ANOVAsconditions (experimental vs. control) $\times$ familiar stimulus (prototype vs. nonprototype), performed on the individual scores from the first three and last three familiarization trials - did not reveal any reliable differences $[F(1,60)<1.00, p>.10$, in all instances $]$.

Difference scores. Difference scores were again computed by subtracting the mean looking time for the last three familiarization trials from the mean looking time for the first three postshift trials for each subject. The mean difference scores are displayed in Table 3. As in Experiment 1 , only experimental infants who were familiarized with the nonprototype showed a large increase in their looking response. An ANOVA-conditions (experimental vs. control) $\times$ familiar stimulus (prototype vs. nonprototype), performed on the individual difference scores-revealed significant main effects for conditions $[F(1,60)=5.80, p<.01]$ and familiar stimulus $[F(1,60)=$ $4.39, p<.05]$. As in Experiment 1, the expected interaction between conditions and familiar stimulus was not significant $[F(1,60)=1.98, p>.10]$.

Importantly, however, individual comparisons again revealed evidence of internal category structure. In particular, experimental infants familiarized with a nonprototype showed a reliably greater difference score than did experimental infants who were familiarized with a prototype $(p<.01)$. In addition, experimental infants familiarized with a nonprototype showed reliably greater dishabituation than did their control infants $(p<.01)$, whereas experimental infants familiarized with a prototype did not differ reliably from their control infants $(p>$ .10). Thus only the infants who were familiarized with the nonprototype showed evidence of discrimination. Fi-

Table 3

Mean Looking Times (in Seconds) and Standard Deviations for Experiment 2

\begin{tabular}{|c|c|c|c|c|c|c|c|c|}
\hline \multirow[b]{3}{*}{ Familiar Stimulus } & \multicolumn{4}{|c|}{ Preshift Trials } & \multirow{2}{*}{\multicolumn{2}{|c|}{$\frac{\text { Postshift Trials }}{\text { First Three }}$}} & \multirow{2}{*}{\multicolumn{2}{|c|}{ Difference }} \\
\hline & \multicolumn{2}{|c|}{ First Three } & \multicolumn{2}{|c|}{ Last Three } & & & & \\
\hline & $M$ & $S D$ & $M$ & $S D$ & $M$ & $S D$ & $M$ & $S D$ \\
\hline \multicolumn{9}{|c|}{ Experimental Subjects } \\
\hline Nonprototype & 21.16 & 6.44 & 8.04 & 2.76 & 17.99 & 12.19 & 9.95 & 11.1 \\
\hline Prototype & 25.35 & 13.67 & 8.22 & 2.89 & 11.61 & 7.52 & 3.39 & 6.67 \\
\hline \multicolumn{9}{|c|}{ Control Subjects } \\
\hline Nonprototype & 21.58 & 7.47 & 7.56 & 1.96 & 10.49 & 5.19 & 2.93 & \\
\hline Prototype & 21.48 & 6.83 & 8.10 & 1.98 & 9.74 & 5.37 & 1.64 & 5.61 \\
\hline
\end{tabular}

*The mean looking times on the first three postshift trials minus the mean times on the last three preshift trials. 
nally, analyses of the difference scores for the last three postshift trials produced no reliable differences $(p>.10)$, although the pattern of difference scores was again like that for the first three trials.

In summary, the basic pattern of asymmetries found in the first experiment for the voiceless category $/ \mathrm{k} /$ was replicated for the voiceless category $/ \mathrm{p} /$. Taken together, the two experiments provide support for the existence of internal category structure for voicing categories by $3-$ 4 months of age.

\section{GENERAL DISCUSSION}

In the present research, we investigated whether the graded internal structure of adult phonetic categories (Kuhl, 1991; Miller \& Volaitis, 1989; Oden \& Massaro, 1978; Samuel, 1982) finds its roots in the early perceptual categories of infants. Recent research by Kuhl and her colleagues (Grieser \& Kuhl, 1989; Kuhl, 1991; Kuhl et al., 1992; and see Polka \& Werker, 1994) has shown evidence of internal category structure for vowels at 6-7 months of age. The present experiments extend this work by providing evidence of such structure for consonant categories as early as $3-4$ months of age. These findings bear on a number of issues, both methodological and substantive.

Consider first a methodological issue that concerns the difference between the procedure we used to test for internal category structure and the one used by Kuhl and her colleagues. Recall that Kuhl's procedure involves comparing discrimination of stimuli in different regions of category space, specifically, in the region of the category prototype and in a nonprototype region. The basic finding, which she terms "the perceptual magnet effect," is that it is more difficult to discriminate stimuli in the region of the category prototype. This is interpreted as a result of a shrinking of the perceptual space around the prototype, which makes discrimination in that region more difficult. Our procedure was based on another kind of asymmetry that has been described in the literaturean asymmetry in perceptual similarity between two stimuli from a given category, a prototype and a nonprototype, depending on which serves as the standard in the similarity task. The empirical finding is that the nonprototype is judged to be more similar to the prototype than the reverse; that is, similarity is judged to be greater when the prototype serves as the standard (Rosch, 1975; see also Krumhansl, 1978, 1979; Tversky, 1977). We found an analogous asymmetry using a discrimination task to indirectly assess similarity: Infants showed greater evidence of discrimination (i.e., presumably judged the stimuli to be less similar) when the nonprototype served as the standard than when the prototype served as the standard. ${ }^{6}$

Both procedures provide evidence for internally structured categories, and, in the case of speech perception, converge to demonstrate structured categories in early infancy. We might speculate that the two procedures are closely related, and, perhaps, that the asymmetry we found is based on a "short-term" magnet effect operating during the course of the experiment. On this view, the infant comes to our procedure with a structured category space centered on the prototype. This structure alone can account for the perceptual magnet effect in Kuhl's paradigm because that involves a comparison of discrimination performance in two different regions of category space, a region centered on the prototype and one displaced from the prototype. But that structure alone cannot account for the asymmetrical pattern of discrimination that we found, because in our paradigm the two stimuli to be discriminated remain constant-they span exactly the same region of category space. What changes is simply which stimulus is presented during familiarization. However, a short-term magnet effect could account for our findings, as follows. When the prototype is the familiarization stimulus, it shrinks the region around the prototype even further; however, when the nonprototype is the familiarization stimulus, it produces no further warping of the space. This results in a smaller perceptual distance between the two stimuli in the former case than in the latter, leading to poorer discrimination when the prototype is the standard.

We should point out, however, that the relation between the two kinds of asymmetries, the one tapped by Kuhl's procedure and the other by the present experiments, appears to be more complex than this analysis would suggest. For example, the type of asymmetry we investigated has been found for many kinds of categories, including line orientation and color (Rosch, 1975), geometric form (Tversky, 1977), and musical tones (Krumhansl, 1979), and the direction of the asymmetry seems to be consistent: Poorer exemplars of a category are judged to be more similar to better exemplars of a category than the reverse. However, recent evidence indicates that the perceptual magnet effect found for speech might not always be found for other categories. In particular, Acker, Pastore, and Hall (1995) have reported that for musical chords, discrimination is actually better, rather than worse, in the region of the category prototype. This suggests that category prototypes might function somewhat differently across domains, and that this difference might interact with the type of task used to assess the underlying structure (see also Krumhansl, 1978, 1979; Tversky, 1977). ${ }^{7}$

We turn now to the substantive implications of our findings, which center on the role played by exposure to the native language in structuring the categories of early infancy. Recall from the introduction that the research on vowels by Kuhl and her colleagues provides evidence not only for internal structure at 6-7 months of age, but also demonstrates that at this age the structure is language specific: Which vowels serve as the category prototypes for infants depends on which vowels serve that function for adults in the native language. This raises the question of whether the initial, language-universal vowel categories of the infant are also internally structured, with nativelanguage exposure serving to fine-tune this structure in accord with the specifics of the native language, or whether the earliest, language-universal vowel categories are unstructured regions of phonetically relevant acous- 
tic space, with internal structure emerging only after exposure to the native language (Kuhl, 1993).

Although we do not yet know the answer to this question for vowel categories, ${ }^{8}$ the present findings address this issue, in at least a preliminary way, for consonant categories. As we described in the introduction, the literature suggests that although exposure to the native language alters vowel categories as early as 6 months of age, it does not appear to alter consonant categories until later, near the end of the 1 st year of life (see Werker, 1994). In particular, research on voicing categories such as those we studied suggests that these categories do not show native-language effects until some time after 6 months of age (Lasky et al., 1975; Werker et al., 1981). The important finding of the present experiments is that at 3-4 months of age, these early perceptual categories are internally structured. It may be, then-at least for consonants - that the graded internal structure of adult phonetic categories finds its roots in early perceptual language-universal categories of the infant that are themselves structured. If so, later exposure to the native language would serve to fine-tune (or perhaps maintain) this early, biologically determined structure in accord with the specifics of its phonetic/phonological properties, rather than lead to its emergence (cf. Aslin \& Pisoni, 1980 ). In order to test this possibility, it will be necessary to determine when internal category structure first appears in early infancy and whether the structure is indeed language universal, and then track alterations in category structure as infants from different language environments are exposed to their native language over the course of development.

Finally, we note that the possible existence of early, language-universal perceptual categories that are internally structured bears on the ongoing controversy in the field concerning the extent to which phonetic perception, and its precursors in infancy, derive from speechspecific processes (see, e.g., Liberman \& Mattingly, 1985) or from more general auditory processes (see, e.g., Diehl \& Kluender, 1989). Kuhl (1991) has found that the perceptual magnet effect for vowels does not appear in monkeys, even though monkeys do partition the phonetically relevant acoustic space of human languages into categories that closely correspond in many cases to those of the young infant. This suggests that, at least for vowels, internal structure, per se, does not derive from psychoacoustic effects based on general mammalian auditory processes. If monkeys also fail to show evidence of internal structure for consonant categories, it is likely that the existence of internal structure in the earliest, languageuniversal categories of infancy may derive not from general psychoacoustic processing, but from specifically phonetic processing.

In summary, the present research provides evidence for the existence of internally structured consonant categories that appear to be analogous to the structured categories of adults as young as 3-4 months of age. Al- though much remains to be learned about the origin and developmental course of these structured categories, it is clear that well before infants acquire the phonetic/ phonological form of their native language, they have the means to map the complex acoustic signal onto categorical representations that resemble adult categories in terms of their within-category gradations, as well as their boundaries. The existence of such structured categories in very early infancy speaks to the strong biological basis for the acquisition of language.

\section{REFERENCES}

Acker, B. E., PASTORE, R. E., \& HALL, M. D. (1995). Within-category discrimination of musical chords: Perceptual magnet or anchor? Perception \& Psychophysics, 57, 863-874.

Aslin, R. N., \& Pisoni, D. B. (1980). Some developmental processes in speech perception. In G. H. Yeni-Komshian, J. F. Kavanagh, \& C. A. Ferguson (Eds.), Child phonology: Perception (Vol. 2, pp. 67-96). New York: Academic Press.

BEST, C. T. (1994). The emergence of native-language phonological influences in infants: A perceptual assimilation model. In J. C. Goodman \& H. C. Nusbaum (Eds.), The development of speech perception: The transition from speech sounds to spoken words (pp. 167-224). Cambridge, MA: MIT Press.

Best, C. T., MCRoberTs, G. W., \& Sithole, N. M. (1988). Examination of perceptual reorganization for nonnative speech contrasts: Zulu click discrimination by English-speaking adults and infants. Journal of Experimental Psychology: Human Perception \& Performance, 14, 345-360.

Carney, A. E., Widin, G. P., \& Viemeister, N. F. (1977). Noncategorical perception of stop consonants differing in VOT. Journal of the Acoustical Society of America, 62, 961-970.

Davis, K., \& KuHL, P. K. (1994). Tests of the perceptual magnet effect for American English $/ \mathrm{k} /$ and $/ \mathrm{g} /$. Journal of the Acoustical Society of America, 95, 2976.

DieHL, R. L., \& KLUENDER, K. R. (1989). On the objects of speech perception. Ecological Psychology, 1, 121-144.

Eimas, P. D., \& Miller, J. L. (1992). Organization in the perception of speech by young infants. Psychological Science, 3, 340-345.

Eimas, P. D., Siqueland, E. R., JusczyK, P., \& Vigorito, J. (1971). Speech perception in infants. Science, 171, 303-306.

Grieser, D., \& KuHL, P. K. (1989). Categorization of speech by infants: Support for speech-sound prototypes. Developmental Psychology, $25,577-588$

HoRowitz, F. D. (1975). Visual attention, auditory stimulation, and language discrimination in young infants. Monographs of the Society for Research in Child Development, 39 (Serial No. 158).

IVERSON, P., \& KuHL, P. K. (1995). Mapping the perceptual magnet effect for speech using signal detection theory and multidimensional scaling. Journal of the Acoustical Society of America, 97, 553-562.

JusczYK, P. W. (1995). Language acquisition: Speech sounds and the beginning of phonology. In J. L. Miller \& P. D. Eimas (Eds.), Speech, language, and communication (pp. 263-301). San Diego: Academic Press.

Krumhansl, C. L. (1978). Concerning the applicability of geometric models to similarity data: The interrelationship between similarity and spatial density. Psychological Review, 85, 445-463.

KRUMHANSL, C. L. (1979). The psychological representation of musical pitch in a tonal context. Cognitive Psychology, 11, 346-374.

KuHL, P. K. (1991). Human adults and human infants show a "perceptual magnet effect" for the prototypes of speech categories, monkeys do not. Perception \& Psychophysics, 50, 93-107.

KUнL, P. K. (1993). Innate predispositions and the effects of experience in speech perception: The native language magnet theory. In B. de Boysson-Bardies, S. de Schonen, P. Jusczyk, P. McNeilage, \& J. Morton (Eds.), Developmental neurocognition: Speech and face processing in the first year of life (pp. 259-274). Dordrecht: Kluwer. 
Kuhl, P. K., Williams, K. A., Lacerda, F., Stevens, K. N., \& LindBLOM, B. (1992). Linguistic experience alters phonetic perception in infants by 6 months of age. Science, 255, 606-608.

Lasky, R. E., Syrdal-Lasky, A., \& Kiein, R. E. (1975). VOT discrimination by four to six and a half month old infants from Spanish environments. Journal of Experimental Child Psychology, 20, 215-225.

Liberman, A. M., \& Mattingly, I. G. (1985). The motor theory of speech perception revised. Cognition, 21, 1-36.

Macmillan, N. A., Goldberg, R. F., \& Braida, L. D. (1988). Resolution for speech sounds: Basic sensitivity and context memory on vowel and consonant continua. Journal of the Acoustical Society of America, 84, 1262-1280.

Massaro, D. W., \& COHEN, M. M. (1983). Categorical or continuous speech perception: A new test. Speech Communication, 2, 15-35.

Medin, D. L., \& Barsalou, L. W. (1987). Categorization processes and categorical perception. In S. Harnad (Ed.), Categorical perception: The groundwork of cognition (pp. 455-490). Cambridge: Cambridge University Press.

MiLLER, J. L. (1977). Properties of feature detectors for VOT: The voiceless channel of analysis. Journal of the Acoustical Society of America, 62, 641-648.

Miller, J. L., Connine, C. N., Schermer, T. M., \& Kluender, K. R. (1983). A possible auditory basis for internal structure of phonetic categories. Journal of the Acoustical Society of America, 73, 21242133.

Miller, J. L., \& Eimas, P. D. (1983). Studies on the categorization of speech by infants. Cognition, 13, 135-165.

MilleR, J. L., \& Volaitis, L. E. (1989). Effect of speaking rate on the perceptual structure of a phonetic category. Perception \& Psychophysics, 46, 505-512.

NosOFSKY, R. M. (1988). Similarity, frequency, and category representations. Journal of Experimental Psychology: Learning, Memory, \& Cognition, 14, 54-65.

Oden, G. C., \& Massaro, D. W. (1978). Integration of featural information in speech perception. Psychological Review, 85, 172-191.

Pisoni, D. B., \& TASH, J. (1974). Reaction times to comparisons within and across phonetic categories. Perception \& Psychophysics, 15, 285-290

Polka, L., \& Werker, J. F. (1994). Developmental changes in perception of nonnative vowel contrasts. Journal of Experimental Psychology: Human Perception \& Performance, 20, 421-435.

REPP, B. H. (1977). Dichotic competition of speech sounds: The role of acoustic stimulus structure. Journal of Experimental Psychology: Human Perception \& Performance, 3, 37-50.

REPr, B. H. (1984). Categorical perception: Issues, methods, findings. In N. J. Lass (Ed.), Speech and language: Advances in basic research and practice (pp. 243-335). New York: Academic Press.

RePP, B. H., \& Liberman, A. M. (1987). Phonetic category boundaries are flexible. In S. Harnad (Ed.), Categorical perception: The groundwork of cognition (pp. 89-112). Cambridge: Cambridge University Press.

Rosch, E. (1975). Cognitive reference points. Cognitive Psychology, 7 , 532-547.

Rosch, E. (1978). Principles of categorization. In E. Rosch \& B. B. Lloyd (Eds.), Cognition and categorization (pp. 28-48). Hillsdale, NJ: Erlbaum.

Samuel, A. G. (1977). The effect of discrimination training on speech perception: Noncategorical perception. Perception \& Psychophysics, 22, 321-330.

Samuel, A. G. (1982). Phonetic prototypes. Perception \& Psychophysics, 31, 307-314.

Schouten, M. E. H., \& van Hessen, A. J. (1992). Modeling phonemic perception. I: Categorical perception. Journal of the Acoustical Society of America, 92, 1841-1855.

Studdert-Kennedy, M., Liberman, A. M., Harris, K. S., \& Cooper, F. S. (1970). Motor theory of speech perception: A reply to Lane's critical review. Psychological Review, 77, 234-249.

Sussman, J. E., \& Lauckner-Morano, V. J. (1995). Further tests of the "perceptual magnet effect" in the perception of [i]: Identification and change/no-change discrimination. Journal of the Acoustical Society of America, 97, 539-552.

Swoboda, P. J., Kass, J., Morse, P. A., \& Leavitt, L. A. (1978). Mem- ory factors in vowel discrimination of normal and at-risk infants. Child Development, 49, 332-339.

Swoboda, P. J., Morse, P. A., \& Leavitt, L. A. (1976). Continuous vowel discrimination in normal and at risk infants. Child Development, 47, 459-465.

TVERSKY, A. (1977). Features of similarity. Psychological Review, 84, 327-352.

Volaitis, L. E., \& Mil.Ler, J. L. (1992). Phonetic prototypes: Influence of place of articulation and speaking rate on the internal structure of voicing categories. Journal of the Acoustical Society of America, 92, 723-735

WAYland, S. C., \& Miller, J. L. (1992, November). The influence of internal phonetic category structure in on-line speech processing. Paper presented at the 33rd annual meeting of the Psychonomic Society, St. Louis.

WERKER, J. F. (1994). Cross-language speech perception: Developmental change does not involve loss. In J. C. Goodman \& H. C. Nusbaum (Eds.), The development of speech perception: The transition from speech sounds to spoken words (pp. 93-120). Cambridge, MA: MIT Press.

Werker, J. F., Gilbert, J. H. V., Humphrey, K., \& Tees, R. C. (1981), Developmental aspects of cross-language speech perception. Child Development, 52, 349-355.

Werker, J. F., \& LALONDE, C. E. (1988). Cross-language speech perception: Initial capabilities and developmental change. Developmental Psychology, 24, 672-683.

WERKER, J. F., \& TEES, R. C. (1984). Cross-language speech perception: Evidence for perceptual reorganization during the first year of life. Infant Behavior \& Development, 7, 49-63.

\section{NOTES}

1. The nature of the representation underlying the internal structure of phonetic categories is currently not known. For example, phonetic categories might be organized around prototypes (summary representations) that are abstracted from the speech signal, or around stored sets of exemplars, presumably weighted for frequency of occurrence (see Medin \& Barsalou, 1987, for discussion). For ease of explication, in the present paper we use the terms prototype and nonprototype to denote "good" and "poor" exemplars of a phonetic category, respectively, remaining agnostic on the precise nature of the underlying representation itself.

2. It should be noted, however, that Iverson and Kuhl (1995) and Sussman and Lauckner-Morano (1995) have provided evidence suggesting that some of the vowel tokens used in Kuhl (1991), including the background stimulus used for the nonprototype group, may not have been perceived by all subjects as variants of $/ \mathrm{i} /$, but as variants of other vowel categories.

3. Note that the prototypical stimulus is $100 \mathrm{msec}$ in one pair, but $110 \mathrm{msec}$ in the other pair. Moreover, the $65 / 110-\mathrm{msec}$ pair has a VOT difference of $45 \mathrm{msec}$, whereas the $100 / 200$-msec pair has a VOT difference of $100 \mathrm{msec}$. These specific pairings were chosen on the basis of the following four constraints: (1) The difference in VOT between stimuli within a pair had to be reasonably large because it is known that small within-category differences in VOT are not discriminable by infants this age (Eimas et al., 1971), and it was not known in advance just how large a within-category difference would be necessary for discrimination (if within-category discrimination occurred at all); (2) we wanted to avoid using stimuli with excessively long VOT values; (3) each stimulus pair had to include a prototypical and a nonprototypical member of the $/ \mathrm{k} /$ category, as defined by the data in Volaitis and Miller (1992); and (4) each prototypical member of the pair had to be near the middle of the best-exemplar range, as defined by the data in Volaitis and Miller (1992).

4. Prior to calculating the mean scores on the first three postshift trials in the two experiments, we eliminated two extreme scores (each on the first postshift trial) and replaced them with the mean looking time for that condition. These scores were more than 3 standard deviations greater than the next highest score. One of these scores was from the experimental group in Experiment 1 familiarized with the 100-msec VOT stimulus; the other was from the experimental group in Experiment 2 familiarized with the $80-\mathrm{msec}$ VOT stimulus.

5 . The individual comparisons reported in this paper were conducted with the Newman-Keuls procedure. 
6. There is one potential case in Kuhl's (1991) design that is analogous to the asymmetry we tested, namely, discrimination of the prototype and nonprototype stimuli along the common vector of her stimulus set by the two groups of infants that were tested, those who heard the prototype as the background stimulus and those who heard the nonprototype as the background stimulus. Data for this specific comparison were not presented in the paper (although an overall analysis of discrimination performance along the common vector did reveal a difference in the two infant groups). Therefore, it is not known whether in Kuhl's data there was an asymmetry in discriminating the prototype and nonprototype stimuli, per se, depending on which was heard as the background stimulus.

7. Although critical, we believe, the distinction between tasks that we draw here was not made by Polka and Werker (1994), who studied the developmental course of a non-native German vowel contrast that is not phonemic in English. English listeners perceive the German vowels as members of the same English category, although one is perceived as a better exemplar of the (English) category than the other. Using a conditioned head-turn procedure, Polka and Werker found evidence that by 6-8 months, English-learning infants also perceive the German vowel contrast in terms of one (English) category, and that this category is internally structured. Polka and Werker discussed their findings in terms of Kuhl's (1991) perceptual magnet effect. However, their design and analysis were based on the type of asymmetry examined in the present experiments, rather than the type reported by Kuhl. That is, they compared discrimination when infants were familiarized with prototypical exemplars and tested on nonprototypical exemplars with the reverse case, rather than comparing discrimination for different regions of category space. Their basic finding in the relevant cases was that infants showed greater evidence of discrimination when the familiarization stimuli were nonprototypical exemplars - a result that parallels the findings of the present experiments. (We should note, however, that the results of the overall study were complex, and that a habituation procedure did not show evidence of either discrimination or internal structure for these vowel categories in infants 6 months of age. The reason for the discrepant results across procedures is not known.)

8. In their study, Polka and Werker (1994) also tested 4-month-old infants on the German vowels using a habituation procedure (see note 7). They found that these infants (unlike the 6-month-olds) could discriminate all vowel pairs. Moreover, the infants perceived the vowel distinctions equally well regardless of whether the prototypical or nonprototypical exemplars served as the familiarization stimuli; that is, no perceptual magnet effect was found. It might be that this lack of asymmetry reflects a lack of internal structure at this early age for the vowel categories. However, it is equally likely that the young infants had not yet been influenced by the parental language and were treating the prototypical and nonprototypical stimuli as exemplars of two languageuniversal vowel categories, in which case the concept of internal structure would not apply. Thus whether infants' vowel categories are internally structured as early as 4 months of age remains to be determined.

(Manuscript received September 11, 1995; revision accepted for publication March 4, 1996.) 